\title{
Bilateral Presence of Two-Rooted Mandibular Second Premolars in an Adult Nigerian Male- A Case Report
}

\author{
Louis Omontuenmen Ibhawoh ${ }^{1 *}$, Ochuwa Audu ${ }^{2}$ \\ ${ }^{1}$ Associate Professor/Consultant, Department of Restorative Dentistry, University of Benin, Benin City/ University of Teaching \\ Hospital, Benin City, Edo State, Nigeria \\ ${ }^{2}$ Senior Registrar, Department of Restorative Dentistry, University of Benin Teaching Hospital, Benin City, Edo State, Nigeria
}

\author{
DOI: $10.36348 /$ sjodr.2021.v06i03.003 \\ | Received: 18.02.2021 | Accepted: 26.02.2021 | Published: 13.03.2021 \\ *Corresponding author: Louis Omontuenmen Ibhawoh
}

\section{Abstract}

For the unsuspecting clinician, a common cause for non-surgical endodontic treatment failure in mandibular premolars is missed roots or canals, as the root canal morphologies of these teeth can sometimes be aberrant. Even in the absence of state-of-the art, three-dimensional imaging techniques, it is prudent clinical approach to have detailed pre-endodontic periapical radiographic assessment of these teeth. Evaluation of sudden changes in the density (fast break) of the root canal space, periodontal ligament contours, root bifurcation, if any, are important points to note, pre-operatively. Bifurcation of mandibular second premolar roots in the mesio-distal axis is very rare and even rarer when it occurs bilaterally. Radiographic exposure of asymptomatic contralateral teeth may also be necessary, to help uncover latent pathologies and evaluate the bilateral presence of anomalies, if any, to justify simple, early restorative intervention to obviate the need for later, more complex root canal treatment.

Keywords: Rare, bilateral, two-rooted, mandibular, second, premolar.

Copyright (C) 2021 The Author(s): This is an open-access article distributed under the terms of the Creative Commons Attribution 4.0 International License (CC BY-NC 4.0) which permits unrestricted use, distribution, and reproduction in any medium for non-commercial use provided the original author and source are credited.

\section{INTRODUCTION}

Predictably successful endodontic treatment requires the complete disinfection, debridement and shaping of the entire root canal systems of teeth, followed by a three-dimensional obturation and final coronal restoration [1]. Studies have noted the high failure rates associated with root canal treatment of mandibular premolar teeth [2-4] and some reasons adduced for this include the occurrence of varied root canal systems, with resultant instrumentation difficulties and missed canals [3, 4].

Mandibular premolars have been traditionally described in endodontic texts as having a single root and a single root canal $[5,6]$. When unusual root and canal configurations or anatomies occur in mandibular premolars, they vary widely in presentation, from a single root with single or multiple root canals, to multiple roots with multiple canals, up to a maximum of four roots and four canals [7-10]. The number of apical foramina in these teeth also vary from single to multiple, sometimes corresponding with the number of canals present [3]. The complexity of the anatomic variations of the mandibular first premolars has led to the consideration of these teeth as the ones most difficult to successfully root-treat [11].

Reports indicate that the incidence of two or more roots in mandibular premolars is still quite rare [7, 12]. Using anatomic studies of mandibular first premolars, it was reported that mandibular second premolars had a single root in $97.9 \%$ of the teeth studied. Two roots were found in only $2 \%$ of the teeth while $0.1 \%$ of the teeth studied had three roots [13]. In another study, a radiographic survey of mandibular second premolars in 600 patients in a South Indian population reported a prevalence of unilateral tworooted second premolars of $1.33 \%$ of the mandibular second premolars while about $0.5 \%$ showed a bilateral presence [7].

Thus, bilateral presence of two-rooted mandibular second premolars has been said to be the rarest anomaly with mandibular premolars [12]. Previously, a few case reports of bilateral two-rooted second premolars in Nigeria have been documented in the literature $[14,15]$. 


\section{CASE RePORT}

The patient was a 21-year old fresh university graduate who had only previously visited the dentist once. He presented on account of spontaneous toothaches from the lower right and left quadrants, posteriorly, worse on the right side. The toothache was associated with headaches, aggravated by mastication and disturbed the patient's sleep. There was no history of any oral swellings. The patient also reported a dull, non-specific and intermittent toothache from the upper left quadrant of his mouth, posteriorly. No significant medical history of note was recorded and the patient was not taking any medication, although he had taken some painkillers for previous toothache episodes.

On intraoral examination, a disto-occlusal Class II carious lesion was noted on the mandibular right first premolar (44) and the tooth was also tender on percussion. The mandibular left second premolar and first molar (35 and 36) also had interproximal carious lesions but were not tender on percussion. Electric pulp tests revealed both teeth 35 and 44 to be totally nonresponsive, while tooth 36 elicited a diminished response, relative to its antimere.

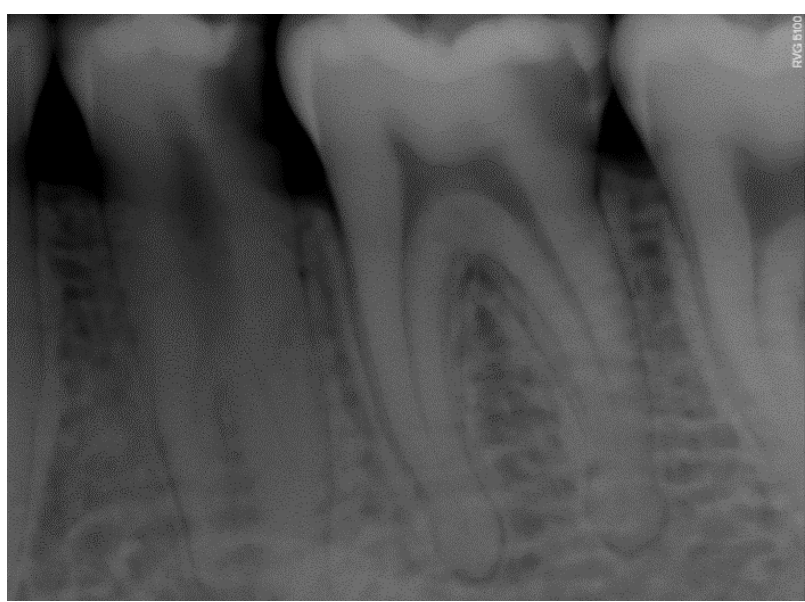

Fig-1: Periapical showing carious lesions and two-rooted mandibular second premolar

Slightly angled diagnostic intra-oral periapical radiographic imaging revealed a coronal radiolucency which appeared to communicate with the pulp space on tooth 44. Tooth 35 and tooth 36 also had coronal radiolucencies which appeared close to the pulp space, with attendant slight widening of their periodontal ligament spaces. The root of tooth 35 was also noted to bifurcate at the mid-root level. Tooth 45 had a similarly bifid root from mid-root, but was caries-free.

A diagnosis of acute apical periodontitis was made for tooth 44, then irreversible pulpitis of 35 and 36. A decision to perform non-surgical root canal treatment of 44, 35 and 36, starting with 44 , was made. Final restorations were going to be post-retained crowns of 35 and 44, with a bonded porcelain crown restoration on 36. Other coincidentally discovered symptom-free carious lesions were also planned for dental restorations.

Tooth 35 and 36 root canal procedures were started one day after starting tooth 44 root treatment. Beginning with tooth 35, conventional access cavities were made on both 35 and 36 with an end-cutting bur, after administered local anesthesia was confirmed to have taken effect. Thereafter, the pulp chamber was unroofed with a round bur, and with the guiding aid of dental loupes, two root canal orifices were discovered on tooth 35-one mesial and the other distal.

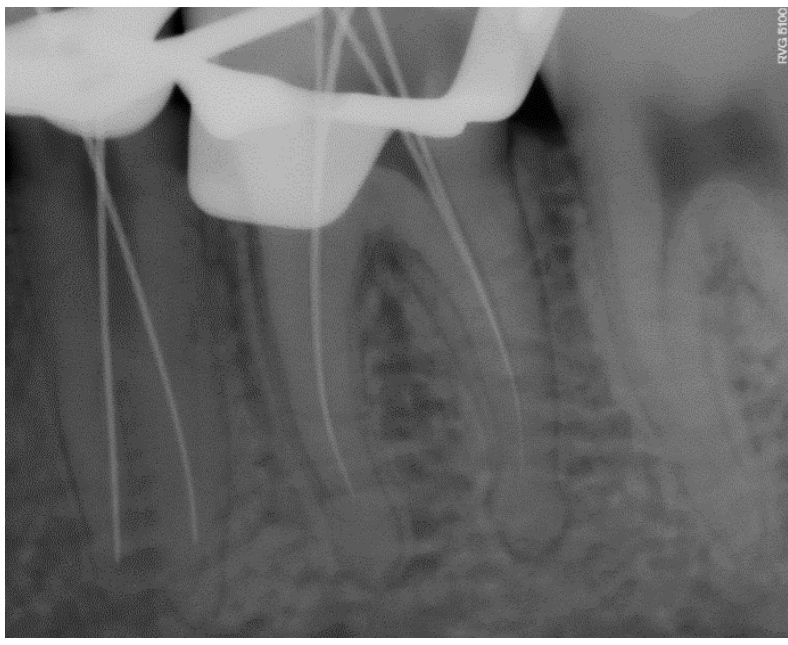

Fig-2: Working length determination

The working length radiographic image was taken with sizes 10 and $15 \mathrm{~K}$-files placed in the mesial and distal canals of tooth 35 . Necrotic pulpal remnants were extirpated from the pulp chamber and root canals of 35 with K-files and copious irrigation with $3.5 \%$ solution of sodium hypochlorite and terminally with normal saline. Further instrumentation could not be carried out as both canals were sclerotic in the apical third.

The canals were dried, filled with EDTA liquid, two cotton pellets were placed over the pulp chamber floor and canal orifices and a temporary access seal of quick setting Zinc oxide eugenol cement was placed. Slight occlusal tooth reduction was done on 35 and 36 to relieve the bite, followed by a 24 -hour recall appointment.

During the second visit, the patient complained of a slight tenderness over 35, during mastication. Further shaping and cleaning of the root canals of 35 and then 36 were done sequentially, using a combination of stainless steel K-files (Mani ${ }^{\mathrm{R}}$, Inc., Tochigi, Japan) for the coronal half of the root canal above the bifurcation and flexible Nickel-Titanium (NiTi) hand-held K-files (NIC ${ }^{\mathrm{R}}$, Shenzhen SuperLine Tech. Co. Ltd, Shenzhen, China) which retain shape memory after pre-curvature, for the two roots below the furcation in the case of the mandibular second 
premolar. Magnification (at 2.5X) using loupes (TaskVision Ltd., USA) was employed during the instrumentation stage. The teeth were thoroughly dried with paper points and their root canals filled with light mixes of non-setting calcium hydroxide. Cotton pellets were placed over the canal orifices on the pulpal floor and a temporary access seal achieved with quick setting Zinc oxide Eugenol cement.

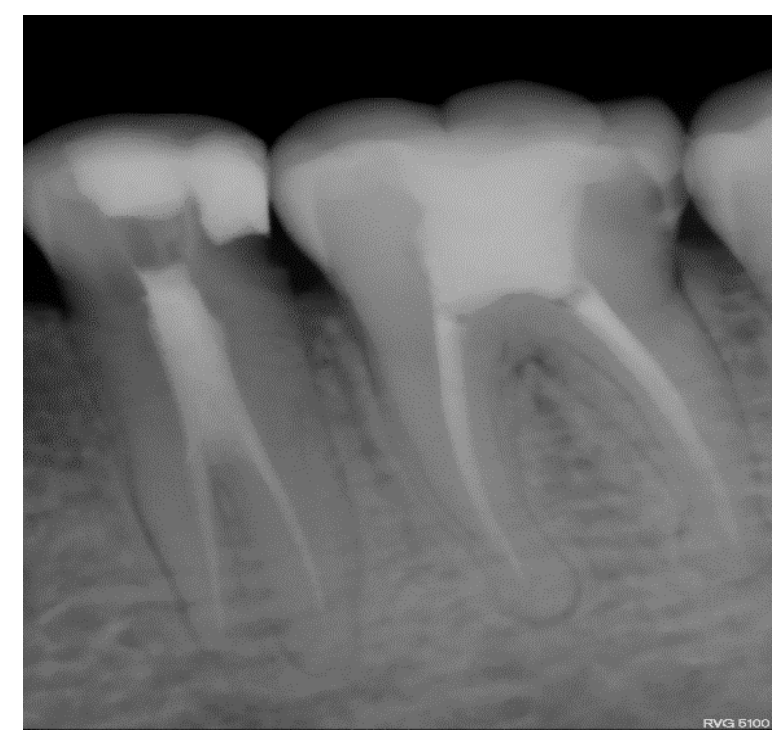

Fig-3: Obturated root canals of 35 and 36

At the third visit, the patient reported no tenderness over either of 35 or 36. Canal obturation was done with gutta percha and a Zinc oxide based sealer (i$\mathrm{ENDO}^{\mathrm{R}}$, by i- DENTAL, UAB Medicinos,Siauliai, Lithuania) using the lateral condensation technique. Posterior Glass Ionomer restorative cement restorations were placed in the teeth, as medium-term restorations since the patient had to travel overseas urgently, where he hoped to receive post-endodontic tooth reinforcement with crown restorations as we had recommended during the treatment planning stage.

\section{THE CONTRALATERAL 44, 45 AND 46}

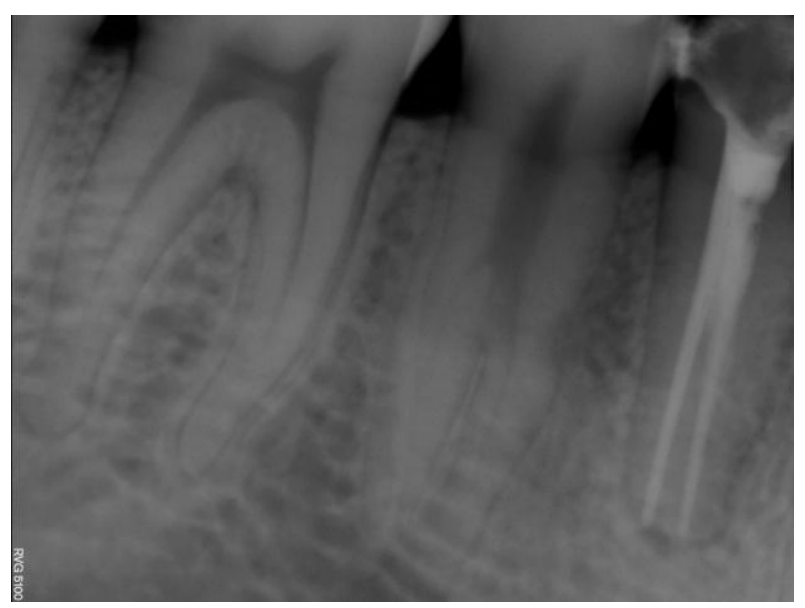

Fig-4

\section{DISCUSSION}

Adequate working knowledge of the various normal root canal morphologies and their possible variations remains an important prerequisite for endodontic treatment success [3]. Mandibular first premolars have been suggested to have the highest failure rates after non-surgical endodontic treatment in all teeth [11]. It cannot be overstated that the possibility of additional root canals being present should always be considered, at treatment onset. Failure to recognize the presence of additional root(s) or canals in any tooth may result in unsuccessful treatment or flare-ups, during and after endodontic treatment [11].

In this case report, diagnostic periapical radiographic images revealed the two roots of the mandibular second premolars beginning at the mid-root level, bilaterally. Additional angled views were taken to exclude the presence of extra roots. It has been reported that aberrant root morphologies can be found bilaterally in almost $90 \%$ of such aberrant cases, and the less common the prevalence of a root anomaly, the more likely it was for it to occur bilaterally [16].

Negotiation and instrumentation of the root canals of tooth 35 was especially challenging, as calcification was noted at the apical thirds of the canals. The two roots found in the second premolars of our patient were also located mesio-distally. This has been reported to be the uncommon presentation of tworooted mandibular premolars, when present $[8,17]$. This case report describes the successful treatment of a tworooted mandibular second premolar, with an emphasis on the mesio-distal arrangement of the two roots and the bilateral presence of this rarity. The paucity of reports of two-rooted mandibular premolars from the Nigerian literature means more studies need to be done on these teeth in Nigerian populations to determine their prevalence especially given the reports $[18,19]$ of their higher prevalence of multi-rooted second premolars seen in African-American populations than in Caucasians as well a $7.8 \%$ prevalence of three canals in mandibular second premolars of African-Americans compared to $2.8 \%$ of Caucasians. Such studies may have an impact on undertaking successful root canal treatment on mandibular second premolars where such anatomically complex cases are encountered.

\section{CONCLUSION}

Mandibular premolars frequently show anatomical complexities which may compromise the chances of undertaking successful root canal treatment. Careful radiographic imaging, treatment planning and modification of canal preparation technique can be very useful in achieving endodontic treatment success. Given that the less commonly an anatomical aberration occurs and then, the greater the chance of its bilateral occurrence, it is advisable to examine closely and explore radiographically, the antimere tooth of any 
carious, two-rooted mandibular premolar and particularly the mandibular second premolar, to initiate timely and less invasive restorative procedures than root canal therapy for that tooth.

\section{REFERENCES}

1. Hariharavel, V. P., Kumar, A. A., Ganesh, C., Annamalai, S., Ramar, K., \& Aravindhan, R. (2014). Root canal treatment of mandibular second premolar with three separate roots and canals using spiral computed tomographic. Case reports in dentistry, 2014.

2. Ingle, J.I., Simon, J.H., Machatou, P. \& Bogaerts, P. (2002). (5th ed.) Outcome of endodontic treatment and re-treatment. In J.I. Ingle, L.K. Bakland LK, (Eds). Ingle's Endodontics. (pp 747757), Hamilton, Ontario. BC Decker Inc.

3. Sharma, D., Sawhney, A., Das, D., \& Srivastava, A. (2017). Mandibular first premolar with two roots: A Case Report. Rama University Journal of Dental Sciences, 4(2):26-29.

4. Jain, S.V., Chandok, M.G., \& Prasad, R. (2013). Endodontic management of a mandibular second premolar with two canals-A case report. Endodontology, 25(2):74-76.

5. Hargreaves, K.M., Cohen, S., \& Berman, L.H. (2011). (10 ${ }^{\text {th }}$ ed.) Tooth Morphology and Access Cavity Preparation. In S. Cohen, H.M. Hargreaves (Eds. Cohen's Pathways of the Pulp. (pp 206-207) St. Louis, Mo. Mosby Elsevier.

6. Wheeler's Dental Anatomy, Physiology, and Occlusion. (2004). ( $8^{\text {th }}$ ed.), M.M. Ash and S. Nelson (Eds.) W.B Saunders. Co., Philadelphia Elsevier. 239-261.

7. Prakash, R., Nandini, S., Ballal, S., Kumar, S.N., \& Kandaswamy, D. (2008). Two-rooted mandibular second premolars: Case report and survey. Indian $J$ Dent Res; 19:70-73

8. Bilateral Mandibular Second Premolar with Mesial and Distal Roots: An Uncommon Feature. Kakkar, P., Singh, A. http://www.livedental.in/articles/endodontics/194bilateral-mandibular-second-premolar-with-mesialand-distal-roots-an-uncommon-feature( Accessed 20/2/2021)
9. Mukhaimer, R.H. (2012). Bilateral mandibular second premolars with three separate roots. Saudi Endodontic Journal, 2(3):156-160.

10. Sachdeva, G.S., Ballal, S., Gopikrishna, V. \& Kandaswamy, D. (2008). Endodontic management of a mandibular second premolar with four roots and four root canals with aid of spiral computed tomography: A case report. Journal of Endodontics, 34:104-107.

11. Sabala, C.L., Benenati, F.W., \& Neas, B.R. (1994). Bilateral root or root canal aberrations in dental school patient population. Journal of Endodontics, 20:38-42.

12. Hiremath, M.C., Srivastava, P. (2015). Bilateral two rooted mandibular second premolar: Report of an unusual case. IOSR-JDMS,14(4):117-120.

13. Cleghorn, B.M., Christie, W.H., \& Dong, C.C. (2007). The root and root canal morphology of the human mandibular first premolar: A literature review. Journal of Endodontics, 33(5):509-516.

14. Oginni, A.O., Olusile, A.O., \& Bamise, C.T. (2002). Root malformation in mandibular premolars. Root malformation in mandibular premolars: an endodontic difficulty- report of two cases. Nigerian Postgraduate Medical Journal, 9: 163-166.

15. Dosumu, O.O., Sulaiman A.O. \& Olasemi, F.S. (2015). Mandibular premolars with multiple roots: a case report. Nigerian Postgraduate Medical Journal, 22; 2: 126-128.

16. Serman, N.J., Hasselgren, G. (1992). The radiographic incidence of multiple roots and canals in human mandibular premolars. International Endodontic Journal, 25: 234-237.

17. Slowey, R.R. (1979). Root canal anatomy: road map to successful endodontics. Dental Clinics of North America, 23:555-573.

18. Trope, M., Elfenbein, L., \& Tronstad, L. (1986). Mandibular premolars with more than one root canal in different race groups. Journal of Endodontics, 12:343-345.

19. Amos, E.R. (1955). Incidence of bifurcated root canals in mandibular bicuspids. Journal of the American Dental Association, 50:70 -71. 